and compared using SPSS. Variables with a $\mathrm{p}$ inferior or equal to 0.05 were considered to be statistically significant.

Results A total of consecutive 89 SLE patients were included: 80 female $(89.9 \%)$ and 9 male $(10.1 \%$ ) (sex ratio $\mathrm{F} / \mathrm{M}$ was 8.9). Mean ages at SLE diagnosis were comparable for men and women respectively $36.9 \pm 15.8$ and. $35 \pm 12.8$ years. History of familiar SLE was more frequent in males than females (22.2\% vs 3.8\%; p=0.078 (Fisher test)). Photosensitivity, cutaneous and renal involvement were significantly more frequent in females $(70 \%$ vs $33.3 \% ; \mathrm{p}=0.027 \%$ and $26.3 \%$ vs $15.6 \%$; $\mathrm{p}=0.078$ respectively). Whereas hemolytic auto immune anaemia and respiratory complications were more frequent in males $(22.2 \%$ vs $1.3 \% ; \quad \mathrm{p}=0.027 \%$ and $55.6 \%$ vs $25 \%$ $\mathrm{p}=0.066$ respectively). There were no differences in articular, cardiac or neurological manifestations. Biological and immunological findings were similar too.

Conclusion It seems that males are more prone to develop SLE when they have familiar history of this disease. They develop more frequently pulmonary manifestations and hemolytic auto immune anaemia. These results should be confirmed by other prospective studies.

\section{PS6:128 INFLUENCE OF ASSOCIATED AUTO IMMUNE DISEASES IN SYSTEMIC LUPUS ERYTHEMATOSUS}

M Kechida, R Mesfar, R Klii, S Hammami, I Khochtali. Internal Medicine and Endocrinology Department, Monastir, Tunisia

\subsection{6/lupus-2018-abstract.171}

Introduction Systemic Lupus Erythematosus (SLE) is a systemic connective disease which can have threatening complications. It can be associated to various other auto immune diseases. Our objective is to know whether the prognosis of SLE is more severe in isolated or in associated SLE.

Patients and methods It's a retrospective study conducted in an internal medicine department. Patients with systemic lupus erythematosus (ACR revised criteria) were included. We compared 2 groups: patients with isolated SLE (ISLE) and patients with associated SLE to another autoimmune disease (ASLE). Variables with a $\mathrm{p}$ inferior or equal to 0.05 were considered to be statistically significant.

Results A total of 89 SLE patients were included: 80 females $(89.9 \%$ ) and 9 males $(10.1 \%$ ) (sex ratio $\mathrm{F} / \mathrm{M}$ was 8.9 ). Mean age was 35.3 years (14 to 72 years). SLE was isolated in 50 patients $(56.1 \%)$ and associated to another autoimmune disease in 39 cases $(43.9 \%)$ as following: sjogren syndrome in 13 patients $(14.6 \%)$, rheumatoid arthritis in 6 patients $(6.7 \%)$, Hashimoto in 5 patients (5.6\%), Basedow in 2 cases $(2.2 \%)$ and SLE was associated to more than an autoimmune disease in 13 cases (11.6\%). Comparative study between ISLE and ASLE showed no significant difference between age onset or sex ratio. Comparing clinical manifestations, patients with ISLE seem to have more cutaneous manifestations and more photosensitivity but without significant difference $(p=0.076$, $\mathrm{p}=0.082$ respectively). Immunological findings showed that anti SSA and anti SSB antibodies were significantly associated to ISLE ( $p=0.007$ and $p=0.013$ respectively). We found no difference in biological findings or SLEDAI score.

Conclusion ASLE seems to develop less specific cutaneous manifestations and to show more frequently positive anti SSA and anti SSB antibodies. When associated to other auto immune diseases SLE doesn't seem to be more severe.

\section{Poster session 7: New drugs and trageted therapy}

\section{PS7:129 SYNERGETIC B-CELL IMMUNOMODULATION WITH RITUXIMAB AND BELIMUMAB COMBINATION TREATMENT IN SEVERE, REFRACTORY SLE}

${ }^{1} \mathrm{~T}$ Kraaij, ${ }^{1}$ SWA Kamerling, ${ }^{1}$ ENM de Rooij, ${ }^{2} \mathrm{PLA}$ van Daele, ${ }^{1} \mathrm{OW}$ Bredewold, ${ }^{3} \mathrm{JA}$ Bakker, ${ }^{4} \mathrm{IM}$ Bajema, ${ }^{5} \mathrm{HU}$ Scherer, ${ }^{5} \mathrm{REM}$ Toes, ${ }^{5} \mathrm{TWJ}$ Huizinga, ${ }^{1} \mathrm{TJ}$ Rabelink, ${ }^{1} \mathrm{C}$ van Kooten, ${ }^{1}$ YKO Teng. ${ }^{1}$ Department of Nephrology, LUMC, Leiden, The Netherlands; ${ }^{2}$ Department of Clinical Immunology, Erasmus MC, Rotterdam, The Netherlands; ${ }^{3}$ Department of Clinical Chemistry and Laboratory Medicine, LUMC, Leiden, The Netherlands; ${ }^{4}$ Department of Pathology, LUMC, Leiden, The Netherlands; ${ }^{5}$ Department of Rheumatology, LUMC, Leiden, The Netherlands

\subsection{6/lupus-2018-abstract.172}

Background Neutrophil extracellular traps (NETs) are autoantigenic DNA strands and potentially give rise to SLE-specific autoantibodies that can deposit in glomeruli. It has been shown that autoantibodies can induce NETs, contributing to the vicious circle of immune activation in SLE. We hypothesised that eliminating autoantibodies can lead to decreased NET induction and thereby ameliorating disease in SLE. Therefore, we designed a proof-of-concept study to eliminate autoantibodies and NET formation through synergetic B-cell immunomodulation with rituximab and belimumab (RTX + BLM) in severe refractory SLE.

Methods We treated patients with severe, refractory SLE in a phase 2 study with RTX+BLM. The primary endpoint assessed reduction of pathogenic autoantibodies and NET induction at 24 weeks. Anti-dsDNA autoantibodies were measured and high sensitivity FACS was performed to assess B-cell subsets. NET induction was measured with $3 \mathrm{D}$ confocal immunofluorescence microscopy.

Results We included 16 patients with severe, refractory SLE of whom 13 had a renal flare. At 24 weeks we observed significant reductions in anti-dsDNA autoantibodies $(\mathrm{p}=0.0004)$. CD19 + B cells were depleted throughout the study $(p=0.0002)$ while plasma cells (PCs) temporarily decreased but returned at week 24 despite persistent depletion of transitional B-cells. Taken together with the observed reductions of autoantibodies and stable total IgG and protective antibodies, there is no reconstitution of autoreactive PCs. Further, we observed excessive NET induction in all patients at baseline which was reduced after 24 weeks $(\mathrm{p}=0.0006)$. In vitro studies elucidated this resulted in reduction of immune complexes by RTX +BLM. Importantly, the beneficial immunological effects translated to amelioration of clinical disease activity: SLEDAI decreased from a median of 18 to 2 ( $\mathrm{p}<0.0001)$. Eleven out of $13 \mathrm{LN}$ patients showed a response $(5$ complete renal responders). The response was achieved while tapering immunosuppressive medication. Treatment was generally well-tolerated. Conclusion The SynBiose study is the first to demonstrate that RTX+BLM ameliorated disease in patients with severe SLE in association with the reduction of pathogenic autoantibodies and immune complex-mediated NET induction. Therefore, RTX+BLM represents a novel treatment concept in SLE. 